\title{
EchoGéo
}

27 | 2014

Structures et armatures urbaines

\section{Environnement et géomatique : des métiers en mutation}

Nathalie Vanara, Cécile Huet, Nicolas Payet, Pierre Pech et Lydie GoeldnerGianella

\section{OpenEdition}

Journals

Édition électronique

URL : https://journals.openedition.org/echogeo/13790

DOI : $10.4000 /$ echogeo. 13790

ISSN : 1963-1197

Éditeur

Pôle de recherche pour l'organisation et la diffusion de l'information géographique (CNRS UMR 8586)

Référence électronique

Nathalie Vanara, Cécile Huet, Nicolas Payet, Pierre Pech et Lydie Goeldner-Gianella, « Environnement et géomatique : des métiers en mutation », EchoGéo [En ligne], 27 | 2014, mis en ligne le 01 avril 2014, consulté le 10 août 2021. URL : http://journals.openedition.org/echogeo/13790 ; DOI : https://doi.org/ 10.4000/echogeo. 13790

Ce document a été généré automatiquement le 10 août 2021.

EchoGéo est mis à disposition selon les termes de la licence Creative Commons Attribution - Pas d'Utilisation Commerciale - Pas de Modification 4.0 International (CC BY-NC-ND) 


\section{Environnement et géomatique : des métiers en mutation}

Nathalie Vanara, Cécile Huet, Nicolas Payet, Pierre Pech et Lydie GoeldnerGianella

\section{Les géographes et les métiers dédiés à l'environnement}

1 Les questions environnementales et plus généralement toutes celles touchant au développement durable ont pris une place considérable dans la gouvernance des entreprises, des établissements privés et publics travaillant à l'échelle locale, nationale, européenne et internationale. Les stratégies de ces établissements visent à une meilleure intégration du développement durable dans leur gestion interne et pendant les phases opérationnelles de production, d'autant qu'ils ont maintenant l'obligation d'intégrer dans leurs systèmes d'évaluation (management environnemental global) des éléments aussi divers que le bilan carbone, la performance énergétique ou le respect de la biodiversité. Dans ces conditions, l'environnement est devenu un cadre incontournable des politiques publiques, de l'économie et finalement de la vie des sociétés ; la demande d'expertise dans ce domaine est donc importante.

2 En raison du champ holiste occupé par l'environnement - et comme aucune discipline n'est parvenue à l'intégrer en totalité - nombreuses sont les sciences qui ont développé une réflexion scientifique à partir de laquelle elles forment des professionnels. L'environnement est ainsi abordé en droit, en économie, en philosophie, comme en géographie ou en écologie. Les approches sont diverses parce que :

- la dimension temporelle varie du temps long (en géologie) au temps court (en archéologie) en passant par le temps moyen (en géomorphologie) ;

- les approches peuvent être sectorielles (en climatologie) ou plus transversales (en écologie);

- les approches sont plus naturalistes (géomorphologie, écologie du paysage) ou plus sociétales (géographie humaine, droit, économie, etc.). 
Les Sciences humaines et sociales spécialisées dans l'environnement (et notamment la géographie, discipline s'intéressant aux interactions entre nature et société) préparent les étudiants à une large palette de métiers dans les domaines de la protection et de la gestion de l'environnement mais aussi de l'information et de la communication sur l'environnement. Ces métiers s'exercent dans des structures très variées : associatives, privées (bureaux d'étude, entreprises, auto-entrepreneurs, etc.) ou publiques (services déconcentrés de l'État, établissements publics, collectivités territoriales, établissements publics de coopération intercommunale, etc.). Ces "métiers de l'environnement" sont en forte croissance depuis une décennie ${ }^{1}$ : en France, entre 2004 et 2011, le nombre d'emplois environnementaux (en équivalent temps plein) a augmenté de près de $36 \%$, soit à un rythme annuel moyen de 4,6\%, très supérieur à celui de l'ensemble de l'économie (0,3\%). En 2011, on compte 455600 emplois environnementaux, en équivalent temps plein, dont 321700 dans les éco-activités marchandes des entreprises et environ 114000 (soit un quart) dans les administrations publiques. Ils représentent $1,7 \%$ de l'emploi national. Cette croissance concerne plus particulièrement les domaines de l'eau, des déchets et des énergies renouvelables. Les étudiants qui sortent des cursus « Environnement » proposés par les Sciences humaines et sociales et sont formés à la «Gestion sociétale de l'environnement» ou à l' "Aménagement du territoire et du cadre de vie " connaissent de surcroît une entrée plus aisée et plus rapide dans la vie professionnelle que les étudiants issus des Sciences dites "dures ». Ainsi en 2010, plus de $80 \%$ d'entre eux accédaient à l'emploi à l'issue de leur formation ${ }^{2}$. Cette diversité de métiers s'avère plus large encore lorsque les formations en environnement intègrent une formation à la géomatique.

\section{La géomatique}

4 La géomatique est née de la convergence de la géographie (" géo ») et de l'informatique (« matique »). L'informatique révolutionne les technologies d'acquisition, de stockage, de traitement et de diffusion des données géographiques en permettant leur représentation, leur localisation, leur modélisation et leur analyse grâce à de nouveaux outils facilitant l'émergence de connaissances inédites: un nouveau champ disciplinaire, la géomatique, est alors né. Elle permet la représentation virtuelle, l'étude des interactions et la connaissance des phénomènes localisables afin d'anticiper leurs évolutions, de programmer les actions et d'évaluer leur efficacité. Une des pièces maîtresses de cette discipline est le système d'information géographique, communément appelé SIG. Il assure le traitement des informations contenues dans les bases de données et notamment leur localisation automatisée et leur restitution multimodale, cartographique et statistique. Sa fiabilité repose sur les compétences (informatiques et cartographiques) du géomaticien et sur sa capacité à s'adapter aux différentes thématiques traitées. Il a ainsi pour vocation à construire des données géographiques à partir d'informations issues de différents savoirs disciplinaires.

5 L'environnement et le paradigme du développement durable impliquent maintenant tous les secteurs professionnels de l'environnement; d'importants enjeux juridiques et économiques s'y associent. Le contexte est favorable aux projets transversaux et pluridisciplinaires. Des acteurs, aux compétences diverses, sont désormais amenés à se parler, à travailler et à se comprendre pour gérer efficacement un territoire. Représenter les enjeux de cet espace devient incontournable; le géomaticien utilise 
donc l'outil SIG qui, à partir de données brutes, propose des représentations cartographiques et des modélisations objectives, compréhensibles par tous. La possibilité de travailler sur des données multidimensionnelles (multi-échelles, multidates, multi-couches/multi-thématiques) spatialisées permet de traduire la complexité d'une problématique territoriale et de restituer de manière simple des résultats qualitatifs et quantitatifs utiles à la prise de décision. Pouvoir croiser des données et/ou des modèles classés par période de temps permet d'effectuer des simulations de phénomènes naturels ou anthropiques pour une meilleure anticipation des évolutions possibles.

6 Par son rôle fédérateur et médiateur, le SIG est donc un outil indispensable à la compréhension, la gestion et le management de l'environnement.

\section{Le rapprochement entre géomatique et environnement : une réponse aux enjeux scientifiques et sociaux contemporains}

7 Plusieurs évolutions sociales ou environnementales expliquent le recours croissant à la géomatique dans les métiers de l'environnement. Ces évolutions relèvent tant d'une nécessaire réponse face aux incertitudes et enjeux environnementaux que d'une transformation en profondeur des sociétés avec notamment l'augmentation des contraintes juridiques et économiques.

\section{La géomatique, une réponse aux incertitudes et aux enjeux environnementaux}

8 Certains métiers s'intéressant à l'environnement ont été modifiés favorablement par la géomatique. Trois domaines au moins en attestent: 1/ la gestion des risques, 2/ les études préalables et études d'impact avant la construction d'infrastructures ou de zones d'aménagement, 3/ la compréhension de l'organisation et des dynamiques du vivant.

\section{La réglementation de la gestion des risques}

Elle est élaborée essentiellement à partir de la loi de 1995, dite loi Barnier. Elle institue les Plans de prévention des risques (PPR) discrétisés en fonction de la nature d'un aléa spécifique: PPR Inondation, PPR Avalanche, PPR Technologique par exemple. Le principe est celui du zonage des aléas et des vulnérabilités, puis de la définition de périmètres de risques probables. Ainsi, les études techniques préalables qu'elles soient géotechniques ou hydrauliques permettent d'élaborer les périmètres d'inondabilité sachant que la loi de 1995 considère que le niveau d'inondation d'une crue de récurrence centennale définit le risque majeur d'inondation. La cartographie s'appuie depuis lors sur l'utilisation de la géomatique qui peut intégrer les données géoréférencées et permettre de gérer les éléments du PPR non seulement dans sa phase d'élaboration mais aussi dans sa phase d'exploitation notamment comme document réglementaire d'urbanisme, en particulier dans les Plans locaux d'urbanisme (PLU). Ce document présente l'avantage de pouvoir rapidement évoluer grâce à l'utilisation de bases de données intégrées à un système d'information géographique; il devient 
interactif quand il intègre d'autres contraintes réglementaires, comme des Servitudes d'utilité publique localisées dans les périmètres du PPR. La loi de 2003, dite loi Bachelot est venue renforcer cette réglementation. En particulier, elle institue, pour les risques technologiques, les secteurs susceptibles de faire l'objet de compensations financières dans le cas de décisions d'expropriation ou de délaissement.

\section{La loi de 1976 sur les études d'impact}

10 Elle préfigure ce qui va être développé dans les années suivantes. En accompagnement d'un projet d'aménagement d'envergure comme une autoroute, une voie ferrée, une carrière, une zone d'activité, sont mis en évidence les impacts environnementaux du chantier puis de l'infrastructure dans sa phase d'exploitation. Le diagnostic environnemental repose sur une batterie de données géoréférencées, et c'est pourquoi toute étude d'impact s'appuie désormais sur un SIG.

\section{L'écologie du paysage}

La révolution scientifique qu'a constituée l'écologie du paysage a été déterminante et a des répercussions dans de nombreux domaines. Des notions comme celles de corridor ou de fragmentation, tout comme celle des hydrosystèmes, ont été transférées dans de nombreux domaines scientifiques, mais aussi dans le champ du discours des politiques publiques, avec par exemple en France les TVB, trames vertes et bleues. Dans ce contexte, l'intégration de la géomatique à l'écologie du paysage a permis l'émergence d'un nouveau champ de compréhension des dynamiques de l'organisation du vivant et de ses milieux de vie.

\section{La géomatique, une réponse aux évolutions sociales et technologiques}

\section{Évolutions sociales}

12 Concernant les transformations sociétales, le recours à la géomatique est principalement lié à la multiplication des acteurs impliqués dans les questions environnementales avec notamment la participation croissante de la société civile. Les problématiques environnementales peuvent d'ailleurs être considérées comme les moteurs de la participation sociale car elles nécessitent d'intégrer de nouvelles formes de connaissances face aux incertitudes grandissantes, de mieux partager les responsabilités entre acteurs, de trouver des solutions à des échelles locales multiacteurs pour mener des politiques acceptables et, si possible, acceptées par tous (C. Bouni, Asca). En France, la participation sociale a fortement progressé durant les quarante dernières années. Initiée en 1976 par la loi de Protection de la nature qui crée les études d'impact et l'obligation d'information en la matière, elle est renforcée en 1983 par la loi Bouchardeau qui accorde plus d'importance à l'opinion sociale dans le cadre des enquêtes publiques. La conférence internationale de Rio, en 1992, conforte ces principes par l'incitation à la participation de tous les citoyens au traitement des questions environnementales. Par la suite, les circulaire Bianco, en 1992, s'intéressent aux grands projets d'aménagement et à la question de leur opportunité et la loi Barnier, en 1995, à l'organisation de débats publics par une commission nationale ad hoc, devenue indépendante en 2002 (loi sur la Démocratie de proximité). À l'échelle 
internationale, la convention d'Aarhus, en 1998, renforce les notions de libre accès à l'information et de participation de tous aux décisions environnementales. Désormais, la gouvernance à six telle qu'elle est actuellement pratiquée, associe à l'État et aux collectivités territoriales, les syndicats, les entreprises, les associations environnementales ou de consommation et les grands élus. Dans ce contexte, la géomatique est de plus en plus fréquemment utilisée. Par exemple, elle permet dans le cas d'un aménagement soumis à un débat, une localisation des enjeux étudiés ou discutés collectivement; dans le contexte d'une discussion d'un PPR, une superposition des aléas et des enjeux communaux. Les cartes, qui font le plus souvent réagir la société civile, sont celles qui associent aux zones d'habitat de nouveaux enjeux ou aménagements susceptibles d'induire des évolutions dommageables ou contestables, collectivement ou individuellement. Ainsi, pour la société, la géomatique se révèle être autant un outil d'information et de participation qu'un nouveau moyen de contestation.

\section{Évolutions technologiques}

13 Depuis la fin du $\mathrm{XX}^{\mathrm{e}}$ siècle, le recours croissant à la géomatique s'explique aussi par l'utilisation massive de l'image grâce à l'émergence des Nouvelles technologies de l'information et de la communication (NTIC). Les NTIC s'immiscent dans la vie professionnelle, citoyenne et personnelle. Qu'elle soit montante ou descendante, verticale ou horizontale, l'information circule en permanence. L'image, vecteur privilégié de la cognition, permet de transmettre un message de manière instantanée et globale. Par son caractère universel, elle est le média idéal pour la représentation des phénomènes spatialisés. Grâce à l'image et aux nouveaux vecteurs de transmission (internet en particulier), l'information géographique devient accessible et partagée par tous. C'est ainsi que sur le portail internet du Museum national d'histoire naturelle sont disponibles quantité de données SIG en libre téléchargement tel l'inventaire national du patrimoine naturel (INPN).

Dans ce contexte, le géomaticien contribue aux échanges entre spécialistes de l'environnement et société. Il met en œuvre ses compétences cartographiques et ses connaissances dans les nouvelles technologies pour produire des informations compréhensibles par tous et les diffuser vers les citoyens, par le biais du traditionnel plan papier ou par celui des récents moyens de la télétransmission (web, smartphone, navigateur GPS, etc.).

\section{La géomatique, une réponse aux contraintes juridiques et économiques}

Depuis quelques dizaines d'années, la multiplication des contraintes législatives et réglementaires dans le domaine de l'environnement s'accompagne d'un accroissement $\mathrm{du}$ nombre de documents tentant de réguler et de gérer l'environnement. Ces documents règlementaires (plans de prévention, schémas directeurs, diagnostics environnementaux, plans d'aménagement et de gestion durable, etc.) sont le plus souvent accompagnés de cartographies issues de traitements sous SIG telles que les simulations de "montée des eaux» pour l'élaboration des PPRI. La composante cartographique de ces documents permet une lecture simple, globale et compréhensible par tous et facilite l'application des normes et des règles. 
16 La géomatique permet aussi de mieux comprendre les enjeux économiques. Par exemple, les études d'évaluation et de mise en concurrence des différents modes de transport de marchandises peuvent désormais tenir compte des impacts sur l'environnement et être rapidement proposés comme le prouvent les actions du Comité de liaison pour les alternatives aux projets de canaux interbassins (CLAC) en 2013. Les études sur les voies navigables, Saône-Rhin ou Rhin-Main-Danube, confrontent respectivement la décroissance et la croissance des transports par voie navigable avec leurs impacts sur l'environnement. Ces comparaisons mettent en balance l'impact positif pour l'économie européenne et l'impact négatif pour l'environnement direct, faune et flore, des zones proches du canal. Pour établir ces comparaisons, le SIG s'avère utile par les simulations d'aménagements et les calculs de distances qu'il permet.

\section{Formations et métiers associant géomatique et environnement}

17 Historiquement, les problématiques environnementales sont issues essentiellement des disciplines universitaires des Sciences de la vie (biologie, écologie) et des Sciences humaines (géographie, droit, économie). Les premières formations dites «environnementales » émanèrent donc logiquement de ces mêmes structures, la composante environnementale étant au départ uniquement une orientation spécifique de la discipline d'origine. Depuis une dizaine d'année, l'environnement tend à devenir une discipline autonome et des formations spécifiquement dédiées à l'environnement ont été créées.

18 La géomatique, autrefois dénommée "sciences géographiques ", regroupe aujourd'hui un ensemble de techniques de positionnement et de réalisation cartographique, intégrant une part croissante d'informatique et de technologies de l'information. Historiquement, elle n'est enseignée que dans les Grandes écoles spécialisées (École nationale des sciences géographiques, École supérieure des géomètres topographes) voire certains lycées intégrant des classes de BTS spécialisés en topographie.

19 L'association des sciences environnementales et de la géomatique, si elle semble évidente dans ses métiers et ses pratiques professionnelles, ne l'est curieusement pas toujours dans les cursus de formation initiale. Depuis plusieurs années cependant, nombre d'universités proposent dans les maquettes de leurs formations liées à l'environnement des unités d'enseignement de cartographie et de pratique des SIG, selon des volumes horaires variables. De même, les laboratoires de recherche affiliés à ces mêmes universités pratiquent désormais la géomatique quotidiennement dans le cadre de leurs travaux et de leurs publications.

\section{Des formations pluridisciplinaires originales}

Pourtant, les formations qui proposent des activités pédagogiques associant environnement et géomatique de manière fortement intriquée sont encore peu nombreuses. Elles concernent généralement des cursus à caractère professionnel, à l'image des diplômes suivants, tous deux co-habilités par l'université Paris 1 Panthéon-Sorbonne et l'École nationale des sciences géographiques (ENSG). 
21 La licence professionnelle Géomatique et Environnement se décline en trois parcours. Après un socle commun en environnement et en géomatique, les étudiants approfondissent leurs connaissances soit en environnement, soit en conception cartographique, soit en imagerie, afin d'être au plus proche des besoins et techniques diversifiés du monde professionnel.

Le master Développement durable, management environnemental et géomatique (DDMEG) réunit, au sein d'une même promotion, des étudiants issus de quatre filières : géographie, économie, droit et géomatique (cycle ingénieur de l'ENSG). Ce caractère pluridisciplinaire en fait sa principale originalité ; il crée une synergie favorisant un transfert de compétences entre les étudiants. Les compétences géomatiques sont présentes à tous les niveaux du management environnemental. L'objectif est de former ces étudiants, qui présentent des profils variés, aux métiers de l'environnement avec une orientation nettement tournée vers la maîtrise d'ouvrage.

\section{Un exemple d'activité pédagogique}

Dans le cadre des formations à double compétence géomatique et environnement, les étudiants sont préparés à leur futur métier au travers d'ateliers pratiques réalisés en contact étroit avec des professionnels.

Ainsi, en 2012-2013, un groupe de six étudiants du master DDMEG a répondu à une commande de la Direction générale de la sécurité civile et de gestion de crise du ministère de l'Intérieur. Le travail consistait à concevoir un outil d'aide à la décision pour la Commission interministérielle "Catastrophes naturelles » dont le rôle est de reconnaître (ou non) l'état de catastrophe naturelle aux communes qui en ont fait la demande auprès de leur préfecture. Les critères permettant d'établir la reconnaissance de catastrophes naturelles sont divers et complexes (topographiques, météorologiques, géologiques, règlementaires - existence ou non d'un PPRI) et les conséquences économiques importantes puisque les citoyens des communes, ayant bénéficié d'un avis favorable, peuvent déclencher une procédure de dédommagement auprès de leurs assurances. Sur ce type de décision à enjeu fort, les données géographiques et les SIG ont un rôle crucial à jouer car ils apportent une information géolocalisée, objective et irréfutable, contribuant à une prise de décision juste et impartiale. Encore faut-il être capable de transcrire le processus de décision dans toute sa complexité en fonctionnalités logicielles. La pluridisciplinarité du groupe d'étudiants (droit, économie, géographie et géomatique) prend alors tout son sens; elle leur permet de mobiliser l'ensemble des compétences enseignées de manière intégrée et globale, et donc de proposer une réponse d'expert à une problématique à enjeux multiples, voire contradictoires.

\section{Les métiers de la géomatique et de l'environnement}

Les métiers qui en découlent se trouvent, à l'image de la géomatique, à la croisée de toutes les disciplines liées à l'environnement.

$\mathrm{Au}$ niveau Licence (Baccalauréat +3 années d'études), les professionnels ainsi formés occupent des postes de cartographes ou gestionnaires de données dans des structures diverses : cellules SIG des parcs et réserves naturelles, bureaux d'études chargés des nouveaux projets d'aménagement ou de construction, services de défense contre les 
incendies ou organismes responsables des inventaires du territoire, des évaluations/ anticipations des catastrophes naturelles ou pollutions, des optimisations de parcours des transporteurs, des implantation d'éolienne, etc.

Au niveau Master (Baccalauréat + 5 années d'études), ils sont recrutés sur des postes de chargé d'études, d'expert en environnement ou d'éco-conseiller. Ils s'intègrent dans des structures publiques (ministères, collectivités territoriales, organismes publics tels que l'ADEME, entreprises) ou privées (bureaux d'étude spécialisés en environnement ou grandes entreprises des secteurs du bâtiment et des travaux publics, de l'eau, des transports, des énergies renouvelables).

\section{Conclusion}

Les compétences géomatiques développées dans le champ de l'environnement représentent des savoirs et des savoir-faire indéniablement en plein essor en France, que ce soit dans le secteur public ou privé. En parallèle, depuis plus de vingt ans, les métiers de l'environnement ont connu un développement incontestable. En témoigne la concurrence légitime qui anime les organismes de formation pour promouvoir l'insertion professionnelle de leurs étudiants, au détriment parfois des cursus proposés par les universités. Ainsi jusqu'en 2007, le concours d'ingénieur subdivisionnaire de la fonction publique territoriale était ouvert à des étudiants issus, non seulement d'écoles d'ingénieur, mais aussi de formations universitaires en environnement ou en aménagement intégrant de la géomatique. En 2007, ce recrutement fut restreint aux seuls élèves issus des écoles d'ingénieurs, possédant ou non des compétences en géomatique. Or, cette discipline mérite, selon nous, une plus grande considération dans les recrutements de cadres de la fonction publique, à la hauteur de la considération déjà acquise dans le secteur privé, notamment lorsque la géomatique est appliquée au champ de l'environnement. Par ailleurs, dans la nouvelle nomenclature des mentions de Masters publiée dans l'arrêté du 4 février 2014, la mention "Environnement » ne figure pas en tant que mention autonome. Elle se trouve diluée au sein de neuf intitulés de mentions (économie, droit, géosciences, agrosciences, géographie, etc.) mais n'apparaît pas dans certaines mentions en sciences sociales (sociologie, philosophie, histoire, psychologie, etc.) et ne peut être associée à la géomatique - autre mention autonome. De ce fait, les formations pluridisciplinaires, telles celles associant l'environnement et la géomatique, risquent de voir décliner leur visibilité et leur reconnaissance, alors qu'elles débouchent sur de nombreux métiers dont on a montré l'importance et les mutations. Les formations combinant géomatique et environnement mériteraient, selon nous, que soit mieux reconnu leur champ pédagogique et scientifique. 


\section{NOTES}

1. CGDD, 2013. Chiffres clefs de l'environnement. Édition 2013, coll. Repères, $64 \mathrm{p}$. http:// www.statistiques.developpement-durable.gouv.fr/fileadmin/documents/Produits_editoriaux/ Publications/Reperes/2013/reperes-chiffres-cle-environnement-2013.pdf

2. CGDD, 2010, L'environnement en France (édition 2010), 150 p.

\section{AUTEURS}

\section{NATHALIE VANARA}

Nathalie Vanara, nathalie.vanara@univ-paris1.fr, géographe, est Maître de conférences-HDR, Université Paris 1-Panthéon-Sorbonne, UMR 8591 LGP et co-responsable (pour la géographie) du M2 DDMEG.

\section{CÉCILE HUET}

Cécile Huet, cecile.huet@ensg.ign.fr, est Ingénieur des travaux géographiques et cartographiques de l'État, enseignante au département de cartographie et d'analyse de l'information géographique, ENSG et co-responsable (pour l'ENSG) du M2 DDMEG.

\section{NICOLAS PAYET}

Nicolas Payet, nicolas.payet@ensg.eu, est enseignant au département de cartographie et d'analyse de l'information géographique, ENSG et co-responsable de la Licence Pro Géomatique et Environnement.

\section{PIERRE PECH}

Pierre Pech, Pierre.Pech@univ-paris1.fr, géographe, est Professeur des universités à l'Université Paris 1-Panthéon-Sorbonne, UMR 8591 LGP et responsable du M2 Bioterre, ancien responsable du M2 DDMEG.

\section{LYDIE GOELDNER-GIANELLA}

Lydie Goeldner-Gianella, lydie.goeldner-gianella@univ-paris1.fr, géographe, est Professeur des universités à l'Université Paris 1-Panthéon-Sorbonne, UMR 8586 PRODIG et co-responsable (pour la géographie) du M2 DDMEG. 